\title{
Life Support Course for Nurses: beyond competency training
}

Yen Tjuin Eugene $\underline{T e o h}^{1}$, PhD, MHS, Jenny Jok Hiok $\underline{\text { Chew }}{ }^{2}$, BcS(Hons), BcHS, Ai Ling Irene $\underline{\operatorname{Lee}}^{1}, \mathrm{MEd}, \mathrm{BN}$, Umadevi Panneer Selvam ${ }^{1}$, MTD, BHS, Fong Chi $\underline{\text { Wee }}^{1}$, MSH, MEd

\begin{abstract}
The Life Support Course for Nurses (LSCN) equips nurses with the resuscitation skills needed to be first responders in in-hospital cardiac arrests. Previous published articles on the LSCN were mainly focused on the development of the LSCN in Singapore, as well as nurses' confidence level, defibrillation experience and outcomes, the perceived barriers faced by nurses and the usefulness of the course. This paper highlights the importance of two key learning methodologies in the LSCN: deep learning and reflection.
\end{abstract}

Keywords: deep learning, Life Support Course For Nurses, reflection, resuscitation

\section{INTRODUCTION}

In Singapore, an estimated 1,900 registered nurses are trained and equipped annually to manage patients with cardiac arrest as key members of the life support team until the patients are transferred to a critical care facility. The Life Support Course for Nurses (LSCN), a brainchild of the National Resuscitation Council (now renamed as the Singapore Resuscitation and First Aid Council [SRFAC]), has been conducted since its inception in 1999. The first LSCN subcommittee in 1999 comprised nurses and doctors from public hospitals, private hospital nursing administrative groups, nursing schools and the Ministry of Health, Singapore.

The Basic Cardiac Life Support (BCLS) course is mandatory for all nurses in Singapore. The LSCN was developed to bridge the gap between the BCLS and Advanced Cardiac Life Support courses. The two-day LSCN aims to train registered nurses to be competent first responders in in-hospital cardiac arrests. It is currently conducted in nursing schools and most public and private hospitals in Singapore.

LSCN instructors play an important role in the programme. Besides conducting theoretical training sessions on 12 life support topics, they also hold group teaching sessions on life support skills such as airway management, defibrillation and megacode. LSCN instructors maintain their competency via their personal clinical practice, by keeping up to date with the latest guidelines and through exchange of best practice in training methodologies.

\section{DEEP LEARNING AND REFLECTION}

Instructors who have an in-depth understanding of learning styles and approaches to learning have the ability to improve or maintain learner interest, as well as accommodate various learning approaches. One such learning approach is 'deep learning', a strategy that aims to achieve desirable learning outcomes with the aid of reflection. Deep learning uses reflective capacities to promote informed, knowledgeable and safe practice. The notion of reflection as a significant concept in education is influenced by a developing awareness among instructors of the need to encourage their learners to be thoughtful, critical and innovative thinkers.

Learners' approaches to learning are defined as an integration of both processes and intentions. It is often argued that learners usually adopt either a deep or a surface approach to learning. Surface learning occurs when learners focus only on facts and details that they believe are important and remember isolated facts without exploring their actual meaning. ${ }^{(1)}$ Learners who adopt a surface learning approach are usually aware of fewer aspects of their learning situations.

By contrast, deep learning occurs when learners not only discover the main facts, but also investigate how the facts relate to each other. ${ }^{(1)}$ The motivation behind deep learning is the desire to understand ideas and seek meaning. Deep learning, according to Majeski and Stover, is "integrative, self-reflective, experiential and self-assessing". (2) The authors further highlighted that deep learning can foster the development of mastery of learning goals, which emphasises the importance of growth and learning as a process. It is opposed to performance or outcome goals. Mastery of learning goals is associated with effort and persistence with challenging tasks. ${ }^{(2)}$ Such goals encourage learners to take on challenging learning tasks and are, therefore, important in the development of deep learning.

Research has shown that there are differences in learning successes between deep and surface approaches to learning. ${ }^{(3)}$ Content learnt through the deep learning approach is retained for a longer time compared with the surface learning approach, where the learner usually retains the information only for the moment. The deep learning approach reaches a deeper level of understanding, whereas the surface approach leads to a surface level of understanding.

The value of reflection as a teaching or learning strategy is increasingly recognised. Reflection is commonly used in our daily lives and has many conceptions and applications.

${ }^{1}$ Nursing Service (Education and Development), Tan Tock Seng Hospital, ${ }^{2}$ School of Health and Social Sciences, Nanyang Polytechnic, Singapore 
However, in the learning context, reflection has a specific meaning - it is a "complex and deliberate process of thinking about and interpreting experience in order to learn from it."(1) Schön proposed two types of reflection: reflection-in-action and reflection-on-action. ${ }^{(4)}$ Reflection-in-action is the process by which the practitioner recognises a new situation and thinks it through while still acting. It is believed that individuals can improve their ability to reflect while in action. Reflection-onaction is the retrospective contemplation of actions that have been taken in order to uncover insights on a particular situation. The reflective practitioner may speculate how the situation might have been handled differently and what other knowledge, skills and attitudes would have been helpful.

Reflection-in-learning has been advocated to bridge the gap between theory and practice. In recent years, educators have included reflective practice in their curriculum, integrating reflective activities in their programme and employing reflection as a form of formal assessment. However, educators often face challenges in teaching and facilitating reflection, as it often lacks a clear definition. Therefore, it is relevant for this paper to discuss and explore reflection as a teaching and learning strategy to enhance deep learning in relation to the LSCN.

During the 1980s and 1990s, most of the literature on reflection-in-learning was based on concepts described by Schön and Dewey. ${ }^{(4,5)}$ Reflection involves deep thoughts - looking back at the situation and casting ideas into the future. As a learning strategy, reflection has to be taught to learners. The aim of teaching the skills of reflection is to develop reflective practitioners who can critically analyse their practice and develop new knowledge. The skills that are necessary to develop reflective practitioners are identified as description, critical analysis, synthesis, evaluation, self-awareness, clinical reasoning skills and problem-solving. ${ }^{(6)}$ This deliberate process of applying theory to practice promotes deep learning. Reflective practice involves a deliberate pause and assumes a higher-level thinking process. Learners can use these processes to examine their beliefs, goals and practices, and in the process, develop deep learning that may lead to changes in their behaviour, skills and attitudes.

Allocating time for reflection during training may be of equal or greater value than time spent at the bedside. Instructors can set aside short periods of time during training to allow learners to gather their thoughts and feelings as a group, as well as consider their actions or the actions of others. Learners can also recall significant events that have happened during scheduled debrief sessions, thus providing an opportunity for structured reflection. These sessions are not for questioning learners on the patient's condition, the pathophysiology or the nursing considerations of treatment. Instead, the educator and students should review a particular experience to enhance awareness and understanding.

\section{LSCN LEARNING AND TEACHING METHODOLOGY}

As responsible trainers, teaching strategies should be initiated to encourage learners to utilise deep learning processes whenever possible. ${ }^{(1)}$ LSCN instructors should discourage surface learning, but instead encourage deep learning by helping the learners to seek meaning, relate what they are learning in one area to another, and to refrain from rote learning, which does not aid understanding. ${ }^{(7)}$

LSCN participants often face challenges in acquiring knowledge and subsequently translating this knowledge to their clinical practice. As novices in resuscitation, they are unable to identify critical information that warrants nursing attention. Hence, these nurses require more exposure in order to recognise and grasp the significance of these clinical situations. As these nurses progress in their learning, they begin to pick up the skill of 'judgement', such as knowledge of when exceptions can be made when performing a skill. Initially, novice nurses need to be given explicit instructions. As they progress to become experts, they will not only rely on work instructions, but also practise discretionary judgement that is demonstrated by the use of scientific rationales and clinical reasoning. The LSCN adopts an educational pedagogy to induct the nurses progressively from basic to more complex competencies over the entire course.

In the teaching context, our LSCN instructors use reflection to allow the learners to relate the theories they have learnt to real-life practice, ultimately leading to deep learning. Reflection-on-action is thought to have generated theories and answered questions that emerge regarding the nature of learning. The usefulness of reflection has become embedded not only in learning literature but also in professional guidelines. Literature on teaching reflection focuses on teaching reflection in the classroom and clinical area. It has been highlighted that both modalities "focus on reflection-on-action as opposed to reflection-in-action" ${ }^{\prime \prime}{ }^{(4)}$ This paper will discuss a few methods used in education to promote deep learning with reflection.

Fundamental to all other methods is a four-step process that can guide reflection. ${ }^{(8)}$ Firstly, learners must identify an event and ask what has happened. Secondly, they will need to analyse and interpret the event, asking themselves questions such as, 'Why did things happen this way?', 'Why did I act the way I did?', 'How did the context affect the experience?' and 'How did past experiences affect the way I reacted?'. Thirdly, learners should ask what they have learnt from the incident, how they can improve and how it might change their future thinking. Lastly, they must consider the implications of their actions by asking what the take-home message is and what the likelihood of productive interactions and learning is.

Critical incident technique has been influential in promoting reflective practice. It involves the identification of behaviours deemed to have been either particularly beneficial or particularly unbeneficial in each situation. Critical examination of incidents usually involves feeling that something could have been improved. Learners are encouraged to record the description of events and their responses to these events, as well as any learning that has occurred, such as how they might have acted differently in a particular situation and the theory/research that might support that situation. ${ }^{(9)}$ Journaling can offer learners an opportunity to become participant-observers of their own learning. Although reflection through journaling is superficial, it is an important first 
step in learning to become reflective. For deeper learning to take place, journals need to be dialogic. Dialogic journals utilise an interactive format and extend a conversation for the purpose of developing self-awareness and understanding of situations.

The LSCN includes the following learning and teaching methodology. Prior to attending the two-day programme, the nurse is required to complete pre-reading of the course contents, which comprise 12 life support topics. These readings are either provided via a hard copy training guide or presented via an electronic learning platform. Pre-recorded practicum videos depicting case-based scenarios or practical skill steps are also included to enable learners to conduct self-directed learning prior to attending the programme. Algorithms from the SRFAC are also provided to learners.

On the first day of the programme, a pre-course test is conducted to allow learners to assess their personal preparation and pre-learnt knowledge. This pre-course assessment will allow learners to self-identify areas of weakness and work towards improving their knowledge and skills in these areas over the next two days. This is followed by either instructor-led lectures or self-directed electronic learning with instructor-led case-based discussions (depending on the training centre). This learning is coupled with station-based practical learning in three main stations: defibrillation, airway management and megacode. The second day of the programme begins with a final theory test, followed by more group practice in the three main practical stations. By the end of the second day, all learners would have undergone practical assessments of all three practical stations and be deemed competent if they fulfil both the theory and practical assessment requirements.

The structured and progressive educational pedagogy of the LSCN is essential to ensure that the learners will increasingly demonstrate greater skill and competence as they are being trained to be competent nurses in resuscitation care. The assessment of clinical performance is vital.

\section{IMPLICATIONS FOR LSCN INSTRUCTORS}

The role of the instructor-educator is essential to the process of developing deep learning and reflective skills in learners. As facilitators of learning, they should adopt a non-authoritarian approach. Instructors should only ask questions to generate discussion and challenge the learner to think in different ways. Input from the educator should be in the form of constructive feedback.
As reflection is usually a new experience for learners, the knowledge that their thoughts and feelings are shared with the instructor can cause some degree of discomfort. Therefore, the instructor should create an environment of mutual trust so that learners will feel safe sharing their feelings and thoughts. In addition, learners are in a vulnerable position during reflection, and may agonise over the possiblity that they may alienate their instructor if they express their fears, questions and thoughts. Therefore, it is imperative that instructors respond to learners' reflection with respect, sensitivity and consideration. Hence, educators play an important role in not only advocating the integration of theory and practice but also developing a reflective approach to education that promotes deep learning.

\section{CONCLUSION}

There is a need to seek new ways and methods of actively involving learners to promote deep learning. Reflection has the potential to engage learners in a dialogue between the theory presented in the classroom environment and the realities of everyday practice. Reflective sessions also offer a means of facilitating development of learners' problem-solving skills, critical thinking and self-awareness. LSCN instructors can move beyond the teaching of theory to using reflection as a tool to enable learners to develop clinical reasoning, which will amplify the impact on learners' practice and patient care.

\section{REFERENCES}

1. Ross GC, Tuovinen JE. Deep versus surface learning with multimedia in nursing education development and evaluation of WoundCare. Comput Nurs 2001; 19:213-23.

2. Majeski R, Stover M. Theoretically based pedagogical strategies leading to deep learning in asynchronous online gerontology courses. Educ Gerontol 2007; 33:171-85.

3. Harper-McDonald B, Taylor G. Expert nurse to novice academic: reflections on the first year of transition from practitioner to academic. Nurs Educ Today 2020 Apr 15. http://doi.org/10.1016/j.nedt.2020.104431. [Epub ahead of print]

4. Schön DA. Educating the Reflective Practitioner: Toward a New Design for Teaching and Learning in the Professions. 1st ed. San Francisco, CA: JosseyBass, 1987.

5. Dewey J. How We Think: A Restatement of the Relation of Reflective Thinking to the Educative Process. Boston, MA: DC Heath, 1933.

6. Atkins S, Murphy K. Reflective practice. Nurs Stand 1995, 9:31-7.

7. Malcolm C, McGirr D. Educational needs and preferred learning approaches of the paediatric palliative care workforce: a qualitative exploratory study. Nurs Educ Today 2020; 89:104417.

8. York-Barr J, Sommers WA, Ghere GS, Montie J. Reflective Practice to Improve Schools: An Action Guide for Educators. Thousand Oaks, CA: Corwin Press, 2001.

9. Nielsen A. Concept-based learning in clinical experiences: bringing theory to clinical education for deep learning. J Nurs Educ 2016; 55:365-71. 